\title{
HIGHLIGHTS
}

GLOMERULAR DISEASES

\section{Anabolic steroid abuse can lead to focal segmental glomerulosclerosis}

Long-term abuse of anabolic steroids can lead to focal segmental glomerulosclerosis (FSGS), according to a recent study.

Although anabolic steroid abuse is known to have adverse effects on the endocrine system - being associated with testicular atrophy, decreased fertility and gynecomastia-and is also associated with changes in blood lipid levels, hepatotoxicity and neuropsychiatric problems, the present study is the first to indicate an association between anabolic steroid abuse and severe renal injury.

The investigators retrospectively identified 10 patients (nine bodybuilders and a professional strong man) who had presented with proteinuria and renal insufficiency and admitted to long-term anabolic steroid abuse. Renal biopsies revealed FSGS in nine patients and glomerulomegaly in one patient.

Clinical follow-up information (mean duration 2.2 years) was available for eight patients. One patient progressed to endstage renal disease within 1 month of biopsy. The other seven patients received renin-angiotensin system blockade and were encouraged to discontinue anabolic steroids and supplements, reduce protein intake and muscle mass, and decrease exercise regimes. After following this advice, serum creatinine levels improved or stabilized and proteinuria decreased in all seven patients. One patient who subsequently resumed his bodybuilding regime and restarted anabolic steroids developed progressive renal insufficiency and a marked increase in proteinuria. "This relapse on rechallenge provides strong evidence of a causal association," notes lead author Vivette D'Agati.

D'Agati and colleagues believe that the etiology of FSGS in such patients is likely to be multifactorial, involving overwork of the kidneys due to elevated BMI and resulting glomerular hyperfiltration,

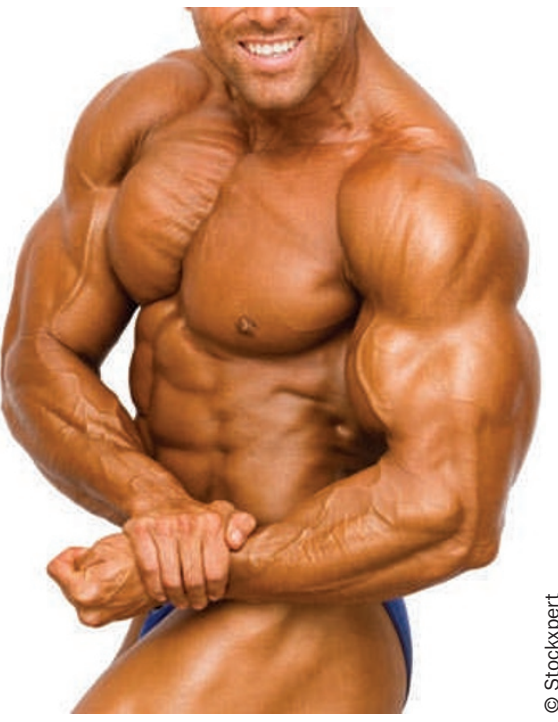

the adverse effects of a high-protein diet and of other supplements, the effects of systemic and exercise-induced hypertension, and potential direct effects of anabolic steroids on glomerular cells.

Rebecca Ireland

Original article Herlitz, L. C. et al. Development of focal segmental glomerulosclerosis after anabolic steroid abuse. J. Am. Soc. Nephrol. 21, 163-172 (2010) 